\title{
EXPLICIT TIME DOMAIN BOUNDARY ELEMENT SCHEME FOR DISPERSION-FREE WAKE FIELD CALCULATION OF LONG ACCELERATOR STRUCTURES*
}

\author{
K. Fujita ${ }^{\ddagger}$, R. Hampel ${ }^{\ddagger}$, W.F.O. Müller ${ }^{\ddagger}$, T. Weiland ${ }^{\ddagger}$, H. Kawaguchi ${ }^{\$}$, S. Tomioka ${ }^{\dagger}$, and T. Enoto ${ }^{\dagger}$ \\ ${ }^{\dagger}$ Graduate School of Engineering, Hokkaido University, Sapporo, Japan \\ ${ }^{*}$ TEMF, Technische Universität Darmstadt, Darmstadt, Germany \\ ${ }^{\$}$ Muroran Institute of Technology, Muroran, Japan
}

\section{Abstract}

This paper introduces a new explicit scheme with a moving window option for wake field calculation of long accelerator structures. This scheme is based on a time domain boundary element method (TDBEM) which uses a retarded Kirchhoff boundary integral equation for interior region problems. As a corollary of this boundary integral equation, our approach allows a conformal modeling of a structure and time domain wake field simulation without numerical grid dispersion errors in all spatial directions. The implementation of a moving window technique in the framework of TDBEM is presented and it is shown that this moving window technique allows to significantly reduce memory requirement of the TDBEM scheme in the short range wake field calculation. Several numerical examples are demonstrated for the TESLA 9-cell cavity and tapered collimators. The results of the new TDBEM scheme are compared with those of finite difference codes.

\section{INTRODUCTION}

The calculation of wake fields and potentials of a short bunch in long structures is an important part in the design of many modern accelerators. So far, Finite Integration Technique (FIT) [1] has been successfully applied for such wake field calculations, and then several improvements [2-5] for geometry approximation and numerical grid dispersion have been done in the framework of the FIT. On the other hand, there are also a few works [6-9] on wake field computations with time domain boundary element methods (TDBEM), which are based on retarded Kirchhoff boundary integral equations. These TDBEM schemes have the following advantages: zero grid dispersion in all spatial directions, conformal modelling of a structure boundary. However, the existing TDBEM schemes still requires very large memory for practical accelerator structures.

In this paper we propose a boundary element scheme with the moving window technique [10] to reduce required memory for practical long structures.

\section{NUMERICAL METHOD}

We consider scattering problems on an interior region $V$ surrounded by the surface $S$ of a perfectly conductor and assume that the bunch is moving in $V$ with the velocity of

*Work partially supported by JSPS Grants-in-Aid of Scientific Research (No. 17540225)

\#fujita@eng.hokudai.ac.jp; fujita@athena.qe.eng.hokudai.ac.jp

05 Beam Dynamics and Electromagnetic Fields light $c$. Then, the total electromagnetic fields $\mathbf{E}$ and $\mathbf{B}$ can be described by a superposition of the bunch self-field $\left(\mathbf{E}_{\text {self }}, \mathbf{B}_{\text {self }}\right)$ and the scattered fields (wake fields) $\left(\mathbf{E}_{\text {wake }}\right.$, $\mathbf{B}_{\text {wake }}$ ). A better approach to deal with this case is to use the scattered field formulation [11]. The wake fields obey the following Kichhoff boundary integral equations for homogeneous material constants $\varepsilon, \mu$ :

$$
\begin{aligned}
& \mathbf{E}_{\text {wake }}(\mathbf{r}, t)=-\frac{1}{4 \pi} \oint_{S}\left\{\left(\mathbf{n}^{\prime} \times \mathbf{E}_{\text {wake }}\left(\mathbf{r}^{\prime}, t^{\prime}\right)\right) \times \nabla^{\prime} \frac{1}{R}\right. \\
& -\frac{1}{R}\left(\mathbf{n}^{\prime} \times \frac{\partial \mathbf{E}_{\text {wake }}}{c \partial t}\left(\mathbf{r}^{\prime}, t^{\prime}\right)\right) \times \nabla^{\prime} R+\left(\mathbf{n}^{\prime} \cdot \mathbf{E}_{\text {wake }}\left(\mathbf{r}^{\prime}, t^{\prime}\right)\right) \nabla^{\prime} \frac{1}{R} \\
& \left.-\frac{1}{R}\left(\mathbf{n}^{\prime} \cdot \frac{\partial \mathbf{E}_{\text {wake }}}{c \partial t}\left(\mathbf{r}^{\prime}, t^{\prime}\right)\right) \nabla^{\prime} R-\frac{1}{R}\left(\mathbf{n}^{\prime} \times \mu \frac{\partial \mathbf{H}_{\text {wake }}}{\partial t}\left(\mathbf{r}^{\prime}, t^{\prime}\right)\right)\right\} d S^{\prime} \\
& \mathbf{H}_{\text {wake }}(\mathbf{r}, t)=-\frac{1}{4 \pi} \oint_{S}\left\{\left(\mathbf{n}^{\prime} \times \mathbf{H}_{\text {wake }}\left(\mathbf{r}^{\prime}, t^{\prime}\right)\right) \times \nabla^{\prime} \frac{1}{R}\right. \\
& -\frac{1}{R}\left(\mathbf{n}^{\prime} \times \frac{\partial \mathbf{H}_{\text {wake }}}{c \partial t}\left(\mathbf{r}^{\prime}, t^{\prime}\right)\right) \times \nabla^{\prime} R+\left(\mathbf{n}^{\prime} \cdot \mathbf{H}_{\text {wake }}\left(\mathbf{r}^{\prime}, t^{\prime}\right)\right) \nabla^{\prime} \frac{1}{R} \\
& \left.-\frac{1}{R}\left(\mathbf{n}^{\prime} \cdot \frac{\partial \mathbf{H}_{\text {wake }}}{c \partial t}\left(\mathbf{r}^{\prime}, t^{\prime}\right)\right) \nabla^{\prime} R+\frac{1}{R}\left(\mathbf{n}^{\prime} \times \varepsilon \frac{\partial \mathbf{E}_{\text {wake }}}{\partial t}\left(\mathbf{r}^{\prime}, t^{\prime}\right)\right)\right\} d S^{\prime}
\end{aligned}
$$

where $\mathbf{r}$ is the observation point in the interior region, $\mathbf{r}^{\prime}$ is the position vector on the surface, $R=\left|\mathbf{r}-\mathbf{r}^{\prime}\right|$, and $t^{\prime}$ is the retarded time defined by $t^{\prime}=t-R / c$. Since the total fields $\mathbf{E}$ and $\mathbf{B}$ are expressed by $\mathbf{E}=\mathbf{E}_{\text {self }}+\mathbf{E}_{\text {wake }}$ and $\mathbf{B}=$ $\mathbf{B}_{\text {self }}+\mathbf{B}_{\text {wake, }}$, the tangential and normal components of $\mathbf{E}_{\text {wake }}$ and $\mathbf{B}_{\text {wake }}$ on the boundary surface are related to the bunch self-fields, real surface current density $\mathbf{K}$ and charge density $\sigma$ as follows:

$$
\begin{aligned}
\mathbf{n} \times \mathbf{E}_{\text {wake }} & =-\mathbf{n} \times \mathbf{E}_{\text {self }}=-\mathbf{M}_{s} \\
\mathbf{n} \times \mathbf{H}_{\text {wake }} & =-\mathbf{n} \times \mathbf{H}_{\text {self }}+\mathbf{K}=\mathbf{K}_{s} \\
\mathbf{n} \cdot \mathbf{E}_{\text {wake }} & =-\mathbf{n} \cdot \mathbf{E}_{\text {self }}+\sigma / \varepsilon=\sigma_{s} / \mathcal{E} \\
\mathbf{n} \cdot \mathbf{H}_{\text {wake }} & =-\mathbf{n} \cdot \mathbf{H}_{\text {self }}=\eta_{s} / \mu
\end{aligned}
$$

where the vector $\mathbf{n}$ is the unit normal to the surface, $\left(\mathbf{M}_{s}\right.$, $\mathbf{K}_{s}$ ) are equivalent surface electric and magnetic current densities and $\left(\sigma_{\mathrm{s}}, \eta_{\mathrm{s}}\right)$ are equivalent surface electric and magnetic charge densities determined by the boundary condition of perfect conductor. The self-fields excite the wake fields indirectly through $\mathbf{M}_{\mathrm{s}}$ and $\eta_{\mathrm{s}}$ in (3). We shall call the TDBEM scheme based on (1) and (2) as Scattered-field Time Domain Boundary Element Method (S-TDBEM) [9].

\section{S-TDBEM system matrix equation}

In this work, we adapt the magnetic field integral equation (MFIE) (2) as a basic equation. Equation (2) is discretized with spatial quadrilateral facets and constant

D05 Code Developments and Simulation Techniques 
time segments $\Delta t$. The surface electric and magnetic current densities located on the edge of boundary elements are linearly interpolated spatially and temporally. The time derivative is approximated by backward finite difference. After performing this discretization procedure, we finally have the following matrix equation [9]:

$$
\left[G_{0}\right]\left[H_{\text {wake }}^{n}\right]=\sum_{i=1}^{L}\left[G_{i}\right]\left[H_{\text {wake }}^{n-i}\right]+\left[H_{\text {ext }}^{n}\right],
$$

where $\left[H^{n}{ }_{e x t}\right]$ is a vector which can be calculated from the boundary integrals of $\mathbf{n} \cdot \mathbf{H}_{\text {wake }}$ and $\mathbf{n} \times \mathbf{E}_{\text {wake }}$ in (2), $\left[H^{n}{ }_{\text {wake }}\right]$ is a vector which consists of the tangential components of the magnetic field $\mathbf{n} \times \mathbf{H}_{\text {wake }}$ on the boundary elements at time step $n$, and $\left[G_{i}\right]$ denotes a coefficient matrix determined by the boundary integral of (2), respectively. By solving the matrix equation (4) at each time step, the electric surface current $\mathbf{K}_{s}$ and charge $\sigma_{\mathrm{s}}$ induced on the structure boundary can be obtained iteratively. Once the boundary values over all time steps have been obtained, the wake fields at any position in the bounded domain can be calculated from (1) and (2). Finally we can compute wake potentials from either the boundary values or the calculated fields $\mathbf{E}_{\text {wake }}$ and $\mathbf{H}_{\text {wake }}$ using the $\mathrm{NCZ}$ indirect integration [8].

\section{Moving Window Technique}

In order to compute the short-range wake potentials in long accelerator structures at low calculation costs, a moving window technique [10] has been used in many Finite Integration Technique codes such as TBCI [1], ECHO [2], PBCI [3], and ROCOCO [5]. For the same purpose, we incorporate the concept of this technique into an explicit version of the S-TDBEM scheme (4) with $c \Delta t$ $<h_{\min }$, where $h_{\min }$ is the minimum mesh size.

We shall consider the S-TDBEM with moving window technique ( S-TDBEM(w) ) on a four-dimensional timespace volume which involves a bunch passing through a long multi-cell cavity as shown in Fig. 1. The basic idea of this technique is that (i) no fields travels in front of an

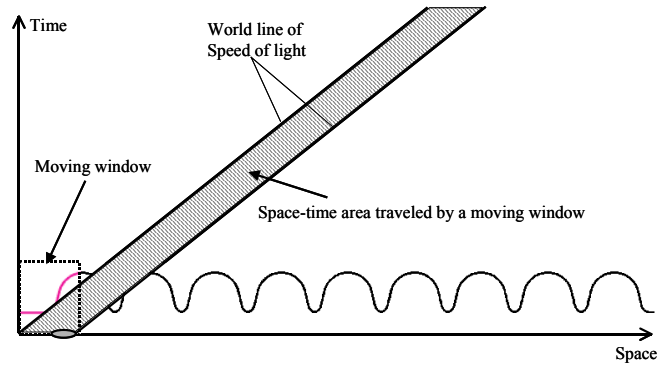

Figure 1. Fields inside a moving window on an accelerating cavity.

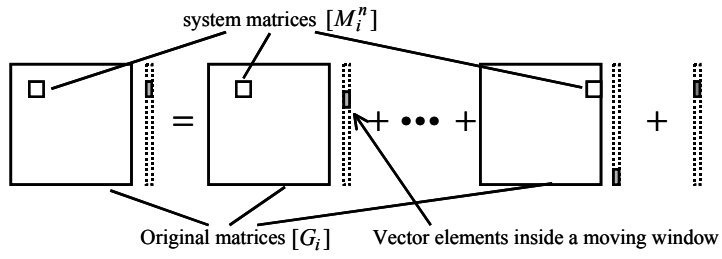

Figure 2. System matrix of S-TDBEM with a moving window

05 Beam Dynamics and Electromagnetic Fields ultra-relativistic bunch and (ii) the fields of a region inside the moving window with speed of light synchronized with the bunch are not affected by any fields that happen behind the window. These two points result from causality and the assumption of rigid bunch. From this reason, we can solve only the fields over the marked space-time volume surrounded by the head and the end of the window. The S-TDBEM basic equation (2) is temporally global in the sense that the fields at present time $t$ depend explicitly on history of the past boundary values inside the window through the boundary integrals including the retarded time. By taking into account this fact and Eq. (4), the S-TDBEM system matrix equation (4) can be reduced as shown in Fig. 2. The reduced matrix equation has many small system matrices $\left[M_{i}^{n}\right]$ determined by the numbers of the boundary value vector elements inside the moving window at each time step $n$. Note that it is necessary for this scheme to reconstruct all of the system matrices by evaluating the complicated boundary integrals in (2) at each time step and the matrix $\left[M_{0}{ }^{n}\right]$ is always diagonal (explicit scheme), and therefore storing the matrices $\left[M_{i}^{n}\right]$ in memory is not needed any more. So, the required memory size of this scheme becomes quite smaller than that of the original TDBEM scheme.

It should be mentioned that this scheme is very timeconsuming due to the reconstruction of the system matrices in every time step. For this, it is possible to save the calculation time by reconstructing the matrices at every $N_{a c c}$ time step and storing the matrices $\left[M_{i}^{n}\right]$ from the $\left(n-N_{a c c}+1\right)$-th step to the $n$-th step in memory. For example, the calculation shown in Fig. 3 (the total number of boundary element along the cross-section $N=20000$, the matrix size stored $M_{\text {matrix }}=500$ x 500 , the total time step $N_{t}=30000=L$ ) takes about 6 hours by the simulation with reconstructing period $N_{a c c}=200$ on a supercomputer with 16 CPUs, while the calculation with every time step matrix reconstructing $\left(N_{a c c}=1\right)$ will take about 50 days by the same supercomputer. The required memory of the S-TDBEM(w) simulation for $N_{a c c}=200$ is about $60 \mathrm{~GB}$ at double precision while the required memory of the S$\operatorname{TDBEM}(\mathrm{w})$ simulation for $N_{a c c}=1$ is less than $100 \mathrm{MB}$. By contrast, for the original S-TDBEM scheme with $\left[G_{i}\right]$ it will be about 96 TB.

Although the above moving window technique for the S-TDBEM scheme (4) has trade-off between calculation time and require memory, dramatic memory saving is realized compared to the conventional TDBEM. This enables us to apply the S-TDBEM(w) for practical accelerator structures as shown in the next section.

\section{NUMERICAL EXAMPLES}

Finally we demonstrate numerical results for two test problems and investigate numerical properties of the S$\operatorname{TDBEM}(w)$ for practical cases by comparison with finite difference codes. 


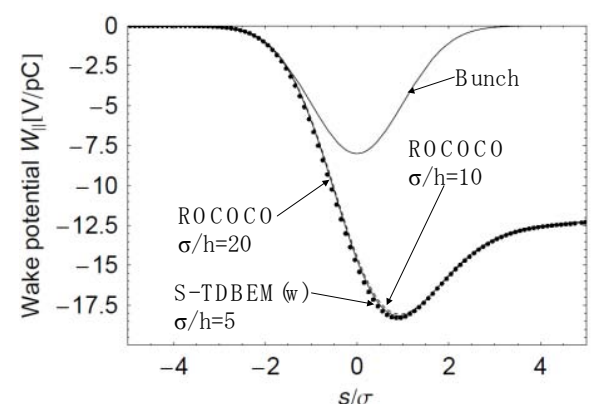

(a) Short range

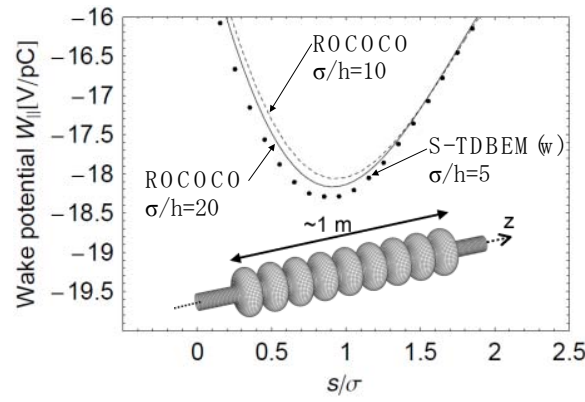

(b) $-0.5 \sigma<s<2.5 \sigma$

Figure 3: Comparison of wake potentials for the TESLA 9cell cavity calculated by the S-TDBEM(w) with the code ROCOCO.

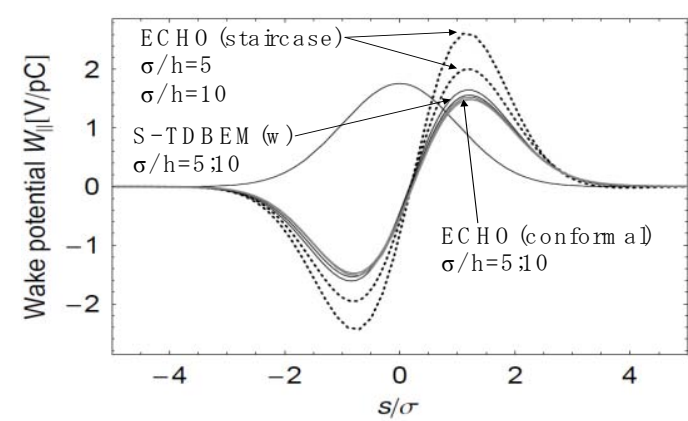

(a) Wake potential

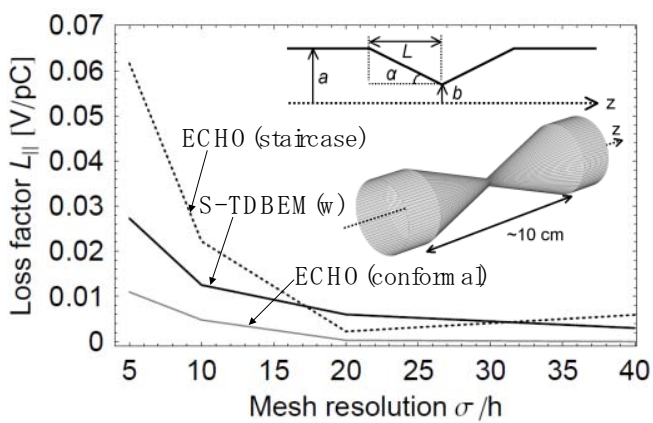

(b) Loss factor

Figure 4. Comparison of numerical results for the tapered collimator computed by the S-TDBEM(w) and the code ECHO.

First, the TESLA 9cell cavity [12] (Fig.3b) is simulated for verifying the zero dispersion property for wake field simulations of a short bunch in long accelerator structures. The ultra-relativistic Gaussian line bunch with RMS length $\sigma=500 \mu \mathrm{m}$ passes along the axis of this structure. Figure 3 shows the comparison of wake potentials computed by the proposed scheme and the code ROCOCO. Unlike the conventional FIT scheme [13], in Fig. 3a the result of the S-TDBEM(w) has no oscillation due to the accumulation of the dispersion error around the bunch tail. In Fig. 3b, even for course mesh $\sigma / h=5$ the proposed scheme gives more accurate result than the ROCOCO result with $\sigma / h=10$.

Second, we deal with a round tapered collimator with geometry parameters $a=19 \mathrm{~mm}, b=1.9 \mathrm{~mm}, L=51 \mathrm{~mm}$ and $\alpha=335 \mathrm{mrad}$ as in Fig. 4b, which is an approximation of the 3D collimator with square aperture installed in the SLAC linac [14]. The convergence property of the proposed scheme is tested for this model. Fig. 4 shows the results of wake potential and loss factor calculated by the S-TDBEM(w) and the code ECHO. The calculation by the S-TDBEM $(w)$ shows much faster convergence than the ECHO calculation with staircase approximation in Fig. 4a. A good convergence of the S-TDBEM(w) is also demonstrated by the numerical results of loss factor in Fig. $4 \mathrm{~b}$. We can conclude that even for practical long structures the S-TDBEM(w) works well as a conformal scheme for dispersion-free wake field calculations.

\section{CONCLUSIONS}

An explicit time domain boundary element scheme with the moving window technique has been presented.
The implementation of the moving window technique has been in detail discussed in the framework of TDBEM. The numerical results show that this scheme allows for conformal and dispersion-free wake field calculations of an ultra short bunch for realistic accelerator structures.

\section{REFERENCES}

[1] T. Weiland, Int. J. Numer. Model. 9 (1996) 295.

[2] I. Zagorodnov, R. Schuhmann, T. Weiland, J. Comput. Phys. 191 (2003) 525.

[3] E. Gjonaj, et. al., in the Proc. ICAP 2006, Chamonix, France, pp.29-34.

[4] M. Kärkkäinen, et. al. in the Proc. ICAP 2006, Chamonix, France, pp.35-40.

[5] R. Hamplel, W. F. O. Muller, T. Weiland, in the Proc. ICAP 2006, Chamonix, France, pp.144-147.

[6] K. Miyata, in the Proc. PAC, Washington, D.C., 1987, pp.1340-1342.

[7] H. Kawaguchi, Eng. Anal. Boundary Elements, vol.27, pp.291-304, 2003.

[8] K. Fujita, H. Kawaguchi, I. Zagorodnov, T. Weiland, IEEE Trans. Nucl. Sci., vol.53, no. 2, pp.431-439, 2006.

[9] K. Fujita, et. al., IEICE Trans. Electron., vol. E90-C, no. 2, 2007.

[10] K. Bane, T. Weiland, in Proc. 12th Int. Conf. on High Energy Accel., Chicago, IL (1983), pp.314.

[11] M. Dehler, Part. Accel., vol.49, pp.105-116, 1995.

[12] TESLA technical design report, Part II, DESY 2001-011, 2001.

[13] R. Hamplel, I. Zagorodnov, T. Weiland, in the Proc. EPAC 2004, Lucerne, Switzerland, pp.2559-2561.

[14] I. Zagordnov, T. Weiland, K. Bane, SLAC-PUB-9985, 2003. 